\title{
OPEN Spatial patterns of lower respiratory tract infections and their association with fine particulate matter
}

\author{
Aji Kusumaning Asri ${ }^{1}$, Wen-Chi Pan ${ }^{2}$, Hsiao-Yun Lee ${ }^{3}$, Huey-Jen Sư ${ }^{4}$, Chih-Da Wu ${ }^{1,5}$ \& \\ John D. Spengler ${ }^{6}$
}

This study aimed to identify the spatial patterns of lower respiratory tract infections (LRIs) and their association with fine particulate matter $\left(\mathrm{PM}_{2.5}\right)$. The disability-adjusted life year (DALY) database was used to represent the burden each country experiences as a result of $L R I s$. $P_{2.5}$ data obtained from the Atmosphere Composition Analysis Group was assessed as the source for main exposure. Global Moran's I and Getis-Ord Gi* were applied to identify the spatial patterns and for hotspots analysis of LRIs. A generalized linear mixed model was coupled with a sensitivity test after controlling for covariates to estimate the association between LRIs and $\mathrm{PM}_{2.5}$. Subgroup analyses were performed to determine whether LRIs and $\mathrm{PM}_{2.5}$ are correlated for various ages and geographic regions. A significant spatial auto-correlated pattern was identified for global LRIs with Moran's Index 0.79 , and the hotspots of LRIs were clustered in 35 African and 4 Eastern Mediterranean countries. A consistent significant positive association between LRIs and $\mathrm{PM}_{2.5}$ with a coefficient of $0.21(95 \% \mathrm{Cl} 0.06-0.36)$ was identified. Furthermore, subgroup analysis revealed a significant effect of $\mathrm{PM}_{2.5}$ on LRI for children (0-14 years) and the elderly ( $\geq 70$ years), and this effect was confirmed to be significant in all regions except for those comprised of Eastern Mediterranean countries.

Lower respiratory tract infections (LRIs), such as pneumonia and bronchiolitis, have been a public health concern for decades due to the severity of illnesses ${ }^{1,2}$. A global study in 2016 has reported that LRIs are the sixth-leading cause of death for all ages and the leading cause of death in children younger than 5 years of age ${ }^{2}$. In total, LRI resulted in 2,377,697 deaths (95\% Uncertainty Interval [UI]: 2,145,584-2,512,809), which includes a high number of fatalities in children younger than 5 years old $(652,572$ deaths, $95 \%$ UI: $586,475-720,612)$ and in elderly people who are at least 70 years old (1,080,958 deaths; $95 \%$ UI: 943,749-1,170,638). Previous studies have confirmed the determinants of LRIs include age, parental and caregiver status, comorbidities (e.g., measles, diarrhea, malaria), and environmental factors ${ }^{3}$. A report released by the United Nations International Children's Emergency Fund in 2016 indicated nearly $50 \%$ of deaths caused by LRIs occur in sub-Saharan Africa ${ }^{4}$. In connection with this issue, several studies related to LRIs especially for children younger than 5 years old have been conducted ${ }^{5-7}$. In addition, a 2016 Global Burden of Disease study asserted emphasizing geographic disparities is the key to reducing fatal outcomes worldwide of LRIs ${ }^{8}$. Therefore, to reduce prevalence of LRIs, more attention should be directed to spatial studies rather than concentrating intervention efforts at the individual level.

In order to correctly identify critical areas of LRIs, a more precise analytic method needs to be applied. Spatial statistical analysis within spatial epidemiology has become indispensable in guiding targeted interventions. Spatial epidemiology studies reach beyond the purview of general spatial statistics by investigating geographic health data with respect to demographic, behavioral, environmental, socioeconomic, and other risk factors ${ }^{9}$. Previous studies have applied spatial analysis to determine health outcome hotspots and have confirmed that these approaches are widely used in epidemiology studies to identify spatial patterns and hotspots of infectious

\footnotetext{
${ }^{1}$ Department of Geomatics, National Cheng Kung University, Tainan 70101, Taiwan. ${ }^{2}$ Institute of Environmental and Occupational Health Sciences, National Yang Ming Chiao Tung University, Taipei 11221, Taiwan. ${ }^{3}$ Department of Leisure Industry and Health Promotion, National Taipei University of Nursing and Health Sciences, Taipei 112, Taiwan. ${ }^{4}$ Department of Environmental and Occupational Health, National Cheng Kung University, Tainan 70101, Taiwan. ${ }^{5}$ National Institute of Environmental Health Sciences, National Health Research Institutes, Miaoli 35053, Taiwan. ${ }^{6}$ Department of Environmental Health, Harvard T.H. Chan School of Public Health, Boston 02115, USA. ${ }^{\circledR}$ email: chidawu@mail.ncku.edu.tw
} 


\begin{tabular}{|c|c|c|c|c|c|c|c|}
\hline Variable & Mean & Std. dev & Min & 25th & Median & 75th & Max \\
\hline \multicolumn{8}{|l|}{ Covariates } \\
\hline Population density (people $/ \mathrm{km}^{2}$ ) & 132.78 & 561.10 & 0.49 & 17.16 & 45.38 & 89.46 & 7249.61 \\
\hline Gender (male \%) & 49.87 & 2.30 & 45.96 & 49.05 & 49.60 & 50.20 & 67.49 \\
\hline Age $0-4$ (years, \%) & 11.46 & 4.68 & 4.03 & 6.97 & 11.23 & 15.15 & 20.99 \\
\hline Age 5-14 (years, \%) & 21.47 & 5.89 & 9.72 & 15.84 & 22.74 & 26.65 & 31.28 \\
\hline Age 15-49 (years, \%) & 50.27 & 4.30 & 40.37 & 47.28 & 49.97 & 53.15 & 67.92 \\
\hline Age 50-69 (years, \%) & 12.40 & 5.59 & 5.51 & 8.06 & 9.88 & 16.70 & 26.67 \\
\hline Age > 70 (years, \%) & 4.40 & 3.30 & 0.60 & 1.84 & 2.90 & 6.71 & 13.02 \\
\hline Economic-income level (categorical variable) & - & - & - & - & - & - & - \\
\hline Alcohol consumption (liters/population/year) & 4.68 & 4.06 & 0.00 & 12.23 & 3.78 & 7.57 & 14.06 \\
\hline Smoking (\%) & 22.14 & 16.26 & 0.00 & 8.25 & 22.70 & 34.35 & 73.40 \\
\hline Healthcare expenditure (\% of GDP) & 6.61 & 2.74 & 0.00 & 4.80 & 6.35 & 8.39 & 17.14 \\
\hline Temperature $\left({ }^{\circ} \mathrm{C}\right)$ & 19.29 & 8.00 & -6.21 & 11.80 & 22.78 & 25.71 & 28.74 \\
\hline Wind speed $(\mathrm{m} / \mathrm{s})$ & 6.37 & 1.48 & 3.09 & 5.27 & 6.41 & 7.41 & 10.41 \\
\hline \multicolumn{8}{|l|}{ Exposures } \\
\hline $\mathrm{PM}_{2.5}\left(\mu \mathrm{g} / \mathrm{m}^{3}\right)^{\mathrm{a}}$ & 17.62 & 14.44 & 0.46 & 7.42 & 14.67 & 23.14 & 67.13 \\
\hline NDVI (normalized difference vegetation index) ${ }^{b}$ & 0.47 & 0.20 & 0.08 & 0.33 & 0.51 & 0.63 & 0.82 \\
\hline \multicolumn{8}{|l|}{ Health outcome } \\
\hline DALY loss due to LRIs (years) & 41.21 & 41.99 & 1.68 & 6.68 & 14.85 & 63.11 & 235.03 \\
\hline
\end{tabular}

Table 1. Baseline characteristics of variables. ${ }^{a}$ Main exposure. ${ }^{b}$ Exposure for subgroup analysis.

diseases $^{10-12}$. In regards to the spatial pattern of LRIs, prior studies conducted in Ethiopia yielded limited research findings because of their reliance on traditional spatial statistics ${ }^{3,13,14}$. In contrast, one study applied Global Moran's I and SaTScan and discovered the acute respiratory infection spatial pattern among children younger than 5 years old in Ethiopia (Moran's $I=0.34)^{15}$. Moreover, LRI clusters were found in the Tigray and Oromia regions ${ }^{15}$. Across various studies, utilization of spatial statistical approaches, such as dot maps, rate maps, Moran's I, and Getis-Ord $\mathrm{Gi}^{*}$, in studying LRIs and other diseases have provided better performance as compared to traditional methods ${ }^{16-19}$.

In addition to the relationship between LRIs and spatial factors, previous studies have noted a strong relationship between LRIs and air pollutants. Beamer applied multivariable regression analyses to investigate the effects of air pollution on health and demonstrated LRIs for which wheezing is a symptom were associated with increased air pollution and, specifically, the presence of 25 different chemical components (OR 1.18, 95\% confidence interval $[\mathrm{CI}] 1.04-1.35)^{20}$. Among the litany of air pollutants, fine particulate matter $\left(\mathrm{PM}_{2.5}\right)$ has been confirmed to be associated with premature death ${ }^{21-23}$. According to the 2010 Global Burden of Disease study, $\mathrm{PM}_{2.5}$ was ranked as one of leading risk factors, contributing to 3.1 million deaths and accounting for $3.1 \%$ of global disability-adjusted life years (DALYs) ${ }^{24-26}$. Moreover, it was estimated for 2015 that 4.2 million (95\% CI 3.7-4.8 million) deaths and 103.1 million (90.8-115.1 million) DALYs were associated with $\mathrm{PM}_{2.5}{ }^{27}$. Fortunately, the negative impacts of air pollutants can be reduced by greenness, leading to a decrease in disease burden and mortality ${ }^{28-32}$. Although the diametric impacts of air pollutants and greenness on health are known, few studies have investigated their relationship with LRIs. Accordingly, in addition to spatial determinants, this study includes air pollutant levels and greenness in its analysis.

To our knowledge, only a few studies have investigated the relationship between LRIs and $\mathrm{PM}_{2.5}$ on a global scale and even fewer have considered levels of greenness exposure. Therefore, this study aims to utilize sophisticated analyses in order to examine the association on a global scale between LRIs and their determinants. By using a country-level database of 183 countries worldwide, this ecological study sought to identify the spatial pattern distribution of LRIs in determining the most critical regions and investigate its linkage with $\mathrm{PM}_{2.5}$ exposure. Global Moran's I and Getis Ord-Gi* were applied for spatial pattern analysis. A generalized linear mixed model coupled with sensitivity tests and subgroup analysis were then estimated to clarify associations with $\mathrm{PM}_{2.5}$ in various circumstances. Since previous studies have confirmed that $\mathrm{PM}_{2.5}$ can increase the burden of LRIs, we assumed $\mathrm{PM}_{2.5}$ would be positive linked to LRIs globally.

\section{Results}

Descriptive statistics. Descriptive statistics for country-level variables at the baseline (data in 2000) are displayed in Table 1 . The mean population density was estimated at 132 people per $\mathrm{km}^{2}$. Moreover, nearly half of the population was male and fell within the age range of 15-49 years. For health behaviors, the annual alcohol consumption and smoking prevalence were $4.68 \mathrm{l} /$ population and $22.14 \%$, respectively. Lastly, the average percentage of healthcare expenditure was $6.61 \%$ of Gross Domestic Product or GDP (SD $=2.74 \%$ of GDP). Regarding exposures, the mean $\mathrm{PM}_{2.5}$ concentration was $17.62 \mu \mathrm{g} / \mathrm{m}^{3}\left(\mathrm{SD}=14.44 \mu \mathrm{g} / \mathrm{m}^{3}\right)$, the mean temperature was $19.29^{\circ} \mathrm{C}\left(\mathrm{SD}=8.00{ }^{\circ} \mathrm{C}\right)$, and the average wind speed was $6.37 \mathrm{~m} / \mathrm{s}(\mathrm{SD}=1.48 \mathrm{~m} / \mathrm{s})$. At the baseline, the mean disability-adjusted life year (DALY) due to LRIs was 41.21 years ( $S D=41.99$ years) per 1000 population. This indicates that among 1000 people, we expect to observe 41.21 years lost to illness or premature death due to LRI. 


\begin{tabular}{|l|l|l|r|l|}
\hline Year & Moran's Index & z-score & p-value & Spatial distribution \\
\hline $2000-2016$ & 0.792 & 12.699 & $<0.001$ & Clustered \\
\hline 2000 & 0.767 & 12.275 & $<0.001$ & Clustered \\
\hline 2010 & 0.770 & 12.392 & $<0.001$ & Clustered \\
\hline 2015 & 0.748 & 12.081 & $<0.001$ & Clustered \\
\hline 2016 & 0.747 & 12.070 & $<0.001$ & Clustered \\
\hline
\end{tabular}

Table 2. Global Moran's I summary of DALY loss due to LRI for each period analyzed.

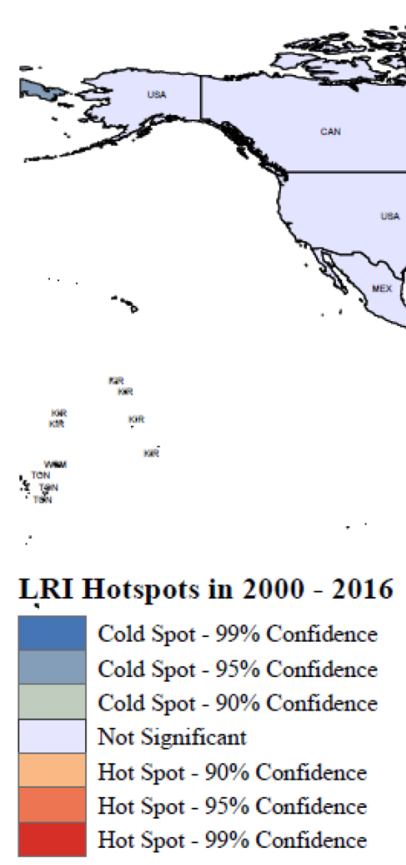

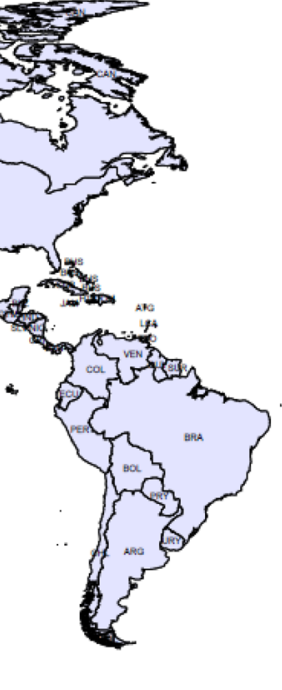

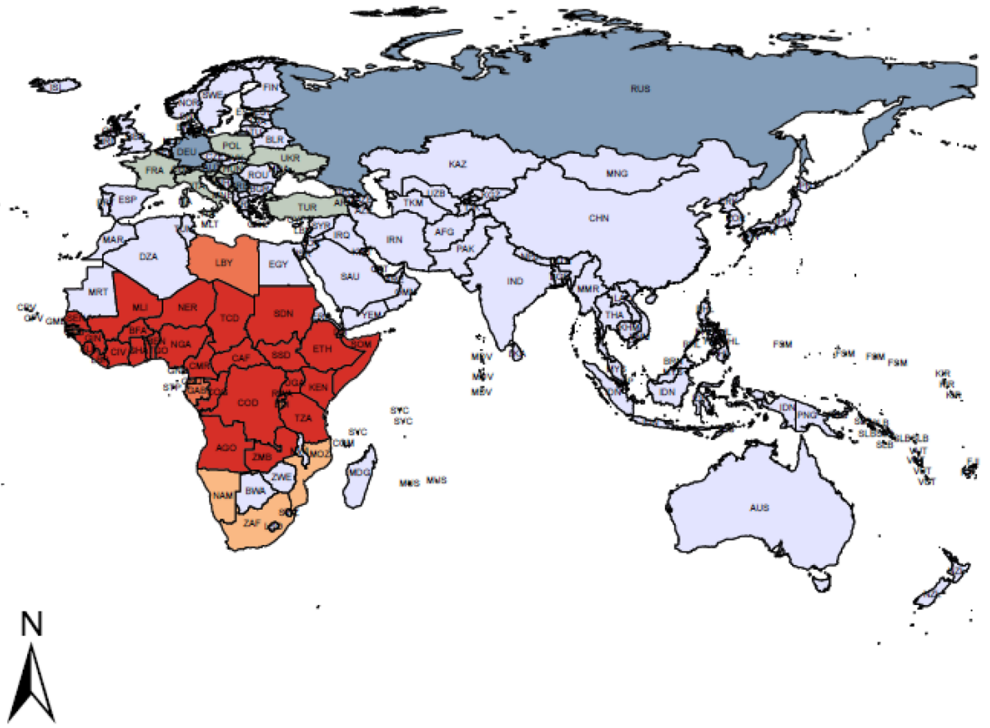

$1: 35.000 .000$

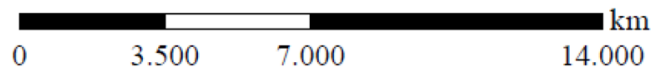

Figure 1. Spatial hot spots and cold spots of DALYs due to LRI.

The trends of DALY due to LRIs are displayed in Supplementary Fig. S4 and they reflect that Africa had the highest burden among all of the regions, though its DALY continually decreased over the study periods.

Spatial pattern and hotspot analysis. Moran's I was utilized in spatial statistics analyses to determine whether or not LRIs are clustered in certain areas. Table 2 lists the results of Moran's I and reflects the spatial autocorrelation of DALY loss due to LRIs during the study periods (i.e., 2000, 2010, 2015, 2016). The positive value of Moran's index means countries have DALY values that are similar to those of the countries surrounding them. The results reveal the value of Moran's I was 0.79 ( $\mathrm{z}$-score $=12.70 ; p<0.001)$, which confirms that a clustered pattern was observed. This result is consistent with visual inspections of hotspot analyses.

With regard to hotspot analysis, the location of clustering was determined using Getis-Ord $\mathrm{Gi}^{\star}$. The hot spot areas were marked red while the cold spot areas were marked blue. As illustrated in Fig. 1, 35 countries in Africa and 4 countries in the Eastern Mediterranean were marked as hotspot areas, which suggests these countries have the highest burden of LRIs as compared to all of the other countries. In contrast, the cold spot areas are dominated by 18 low-DALY countries in Europe. We further examined changes in area, values, and confidence intervals of hot spots due to LRIs by years. These results are displayed in Supplementary Fig. S6a-d. In addition, we used the row-standardized weights matrix for the global measures given earlier to investigate the statistical significance of $\mathrm{z}$-scores $\left(\mathrm{Gi}^{\star}\right)$ assigned to each country, and this revealed the presence and intensity of local clusters of hot spots and cold spots. Confidence intervals of $90 \%, 95 \%, 99 \%$ were reported for each of these in Supplementary Fig. S7a-e.

Association model and sensitivity test. Table 3 lists the results of the statistical association models measuring the significance of the association between $\mathrm{PM}_{2.5}$ and LRIs. After adjusting for pertinent covariates (demographics, socioeconomic status, healthcare status, lifestyle behaviors, and meteorological factors), the main model yielded a significant positive correlation between exposure to $\mathrm{PM}_{2.5}$ and LRIs, with a coefficient of $0.21(95 \%$ CI $0.06-0.36 ; p<0.01)$ in changes in DALY based on an increase of $1 \mu \mathrm{g} / \mathrm{m}^{3} \mathrm{PM}_{2.5}$. This result indicates $\mathrm{PM}_{2.5}$ may increase the disease burden of lower respiratory infections globally. The results of five sensitivity tests with different covariate adjustments (Model 1 to Model 5) and an additional test that eliminated 


\begin{tabular}{|l|l|c|}
\hline Model & Coefficient of PM $_{2.5}(\mathbf{9 5} \%$ confidence interval $[\mathbf{C I}])$ & p-value \\
\hline Main model $^{\mathrm{a}}$ & $0.207(0.058-0.356)$ & $<0.01$ \\
\hline Sensitivity test adjusted for covariates & \multicolumn{2}{|l|}{} \\
\hline Model $1^{\mathrm{b}}$ & $0.204(0.051-0.357)$ & $<0.01$ \\
\hline Model $2^{\mathrm{c}}$ & $0.205(0.052-0.358)$ & $<0.01$ \\
\hline Model $3^{\mathrm{d}}$ & $0.212(0.059-0.365)$ & $<0.01$ \\
\hline Model $4^{\mathrm{e}}$ & $0.211(0.062-0.362)$ & $<0.01$ \\
\hline Model $5^{\mathrm{f}}$ & $0.200(0.051-0.347)$ & $<0.01$ \\
\hline Model $6^{\mathrm{g}}$ & $0.420(0.212-0.627)$ & $<0.001$ \\
\hline
\end{tabular}

Table 3. Association models for LRI and $\mathrm{PM}_{2.5}$, controlled for various covariates. ${ }^{\mathrm{a} C o n t r o l ~ v a r i a b l e s ~ i n c l u d e d ~}$ density of population, sex (\% of male), age, smoking, alcohol consumption, economic status, healthcare expenditure, temperature, and wind speed. ${ }^{b}$ Adjusted for density of population, sex (\% of male), and age. ${ }^{c}$ Adjusted for density of population, sex (\% of male), age, and alcohol consumption. ${ }^{\mathrm{d}}$ Adjusted for density of population, sex (\% of male), age, alcohol consumption, and smoking. ${ }^{\mathrm{e}}$ Adjusted for density of population, sex (\% of male), age, alcohol consumption, smoking, and economic status. ${ }^{\mathrm{f}}$ Adjusted for density of population, sex (\% of male), age, alcohol consumption, smoking, economic status, and healthcare expenditure. ${ }^{g}$ Considered all covariates and eliminated data from Eastern Mediterranean countries.

Eastern Mediterranean countries (Model 6) again confirm the positive association between $\mathrm{PM}_{2.5}$ and LRIs. Model 1 controlled for demographic factors and yielded a $\mathrm{PM}_{2.5}$ coefficient of 0.20 (95\% CI 0.05-0.36). Model 2 controlled for demographics and alcohol consumption and its $\mathrm{PM}_{2.5}$ coefficient was estimated as 0.21 (95\% CI 0.05-0.36). Model 3 controlled for demographics and alcohol consumption as did model 2, however it further controlled for smoking, and its $\mathrm{PM}_{2.5}$ coefficient was 0.21 (95\% CI $0.06-0.37$ ). Model 4 built upon model 3 and included economic status additionally, and its $\mathrm{PM}_{2.5}$ coefficient was 0.21 (95\% CI 0.06-0.36). In model 5, healthcare expenditure was included in addition to all of model 4's covariates, and its $\mathrm{PM}_{2.5}$ coefficient was 0.20 (95\% CI 0.05-0.35). Lastly, model 6 excluded Eastern Mediterranean countries, which yielded a significant and positive $\mathrm{PM}_{2.5}$ coefficient of 0.42 (95\% CI 0.21-0.63).

Subgroup analysis. Figure 2 illustrates the respective association levels between exposure to $\mathrm{PM}_{2.5}$ and LRIs for various subgroups, for instance, by age group, by WHO region, and by WHO regions according to level of greenness exposure. Regarding age groups, the results reflect a positive association between $\mathrm{PM}_{2.5}$ exposure and LRIs in children ( $0-14$ years old) and the elderly ( $\geq 70$ years old). Moreover, there was marginal significance in mature adulthood (50-69 years old). Among all age groups, children younger than 5 years old had the highest positive value for association (coefficient $=0.19 ; 95 \%$ CI $0.05-0.32$ ). When conducting subgroup analysis of the six WHO regions, a significant positive association between $\mathrm{PM}_{2.5}$ and LRI was found for each region except for the Eastern Mediterranean. The overall findings strengthen scientific evidence that the adverse effects of $\mathrm{PM}_{2.5}$ exposure can increase the burden of LRIs in nearly all regions around the world. It is noted the African region had the highest value of $\mathrm{PM}_{2.5}$ (coefficient=1.09; 95\% CI 0.51-1.66), which is consistent with the results of the spatial hotspot analysis (see Fig. 1). Lastly, a subgroup analysis was performed for WHO regions according to their level of greenness. The global results revealed that the effect of $\mathrm{PM}_{2.5}$ was significantly higher in low greenness countries (coefficient $=0.60 ; 95 \%$ CI $0.31-0.90$ ), while countries with high NDVI had marginally significant results. When analyzing results by regions, significant positive associations between $\mathrm{PM}_{2.5}$ and LRIs were found in low NDVI countries in the African, American, European, and Western Pacific regions, where the coefficients were $0.94,0.44,0.38$, and 0.69 , respectively. In contrast, low NDVI countries in Southeast Asia and the Eastern Mediterranean did not display any significance.

\section{Discussion}

Although the associations between global disease burden and mortality from lower respiratory infections and particulate matter have been studied ${ }^{33-36}$, few studies have investigated the spatial pattern of LRIs and analyzed its association with $\mathrm{PM}_{2.5}$ and then linked it to greenness exposure levels. In order to provide a complete and detailed picture of LRIs, this study applied sophisticated analytic methods to examine the spatial pattern and hot spots of LRIs, as well as their association with $\mathrm{PM}_{2.5}$, then linked them to greenness exposure levels at the global scale in analysis. Findings of this study indicate Africa is the region with the highest burden due to LRIs, although its burden trend has gradually been declining. Our findings are consistent with prior studies in which LRIs were estimated to account for $38.6 \%$ of death by infectious disease and $14.9 \%$ of overall deaths in African children ${ }^{37}$. For these reasons, Africa has become a top priority in addressing infectious diseases, especially LRIs ${ }^{38}$. It should be noted the identification of hotspot areas not only identifies areas with the highest rate of disease but also reveals how spatial proximity plays a role in the spread of disease in Africa. In contrast, cold spot areas of LRIs were detected in Europe, suggesting this region has the lowest burden resulting from LRIs. This finding is reinforced by Nowbar's finding that cardiovascular diseases are more common in Europe ${ }^{39}$.

The global model found a significant positive association between $\mathrm{PM}_{2.5}$ and LRIs, even after controlling for covariates. Consistent significant positive associations were also yielded in the ensuing sensitivity tests, and this indicates that a higher level of exposure to country-level $\mathrm{PM}_{2.5}$ is associated with a higher disease burden due to LRIs. Further, in our sensitivity analysis, model 6 indicated a large increase in the estimate of the $\mathrm{PM}_{2.5}$ coefficient 


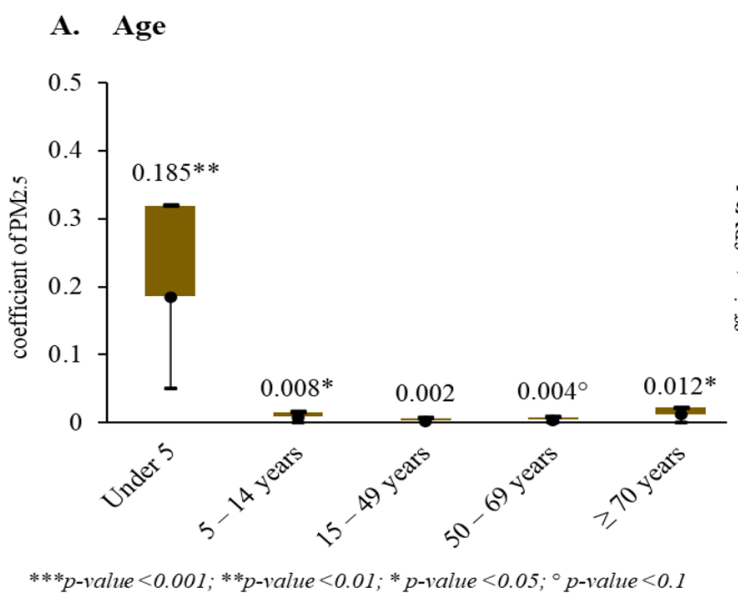

\section{B. Region}

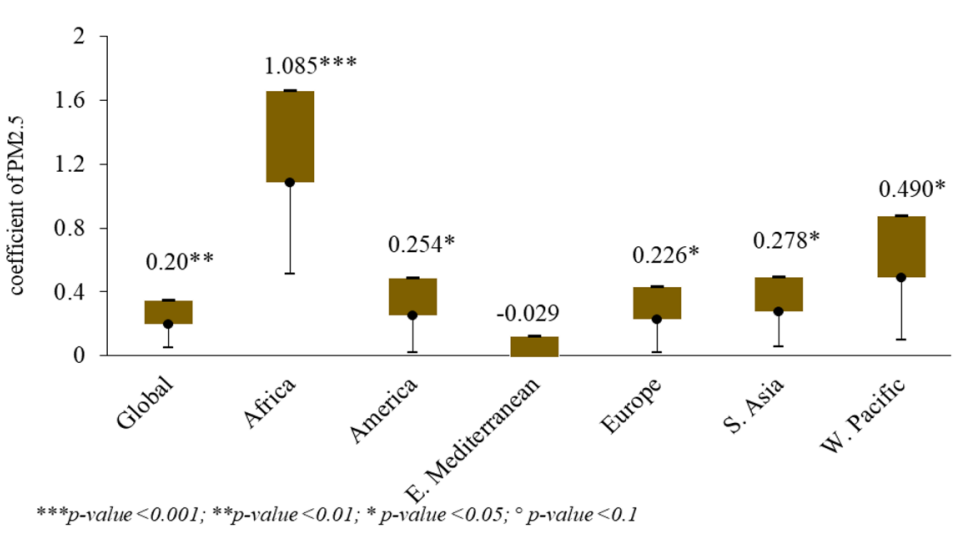

\section{Regions by different levels of greenness exposure}

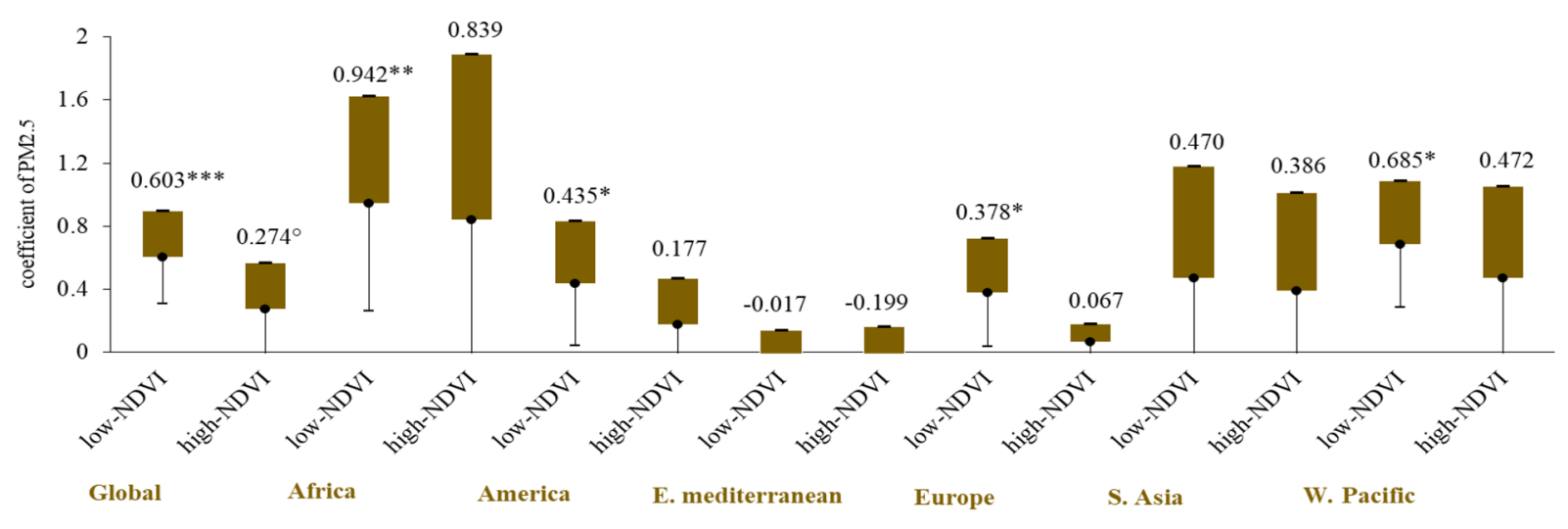

*** $p$-value $<0.001 ; * * p$-value $<0.01 ; * p$-value $<0.05 ;{ }^{\circ} p$-value $<0.1$

Figure 2. Subgroup analysis, stratified by (A) age, (B) WHO region; and (C) WHO Regions by different levels of greenness.

compared to the other five sensitivity models. In this case, a plausible explanation is that in this model we omitted data for Eastern Mediterranean countries that have high $\mathrm{PM}_{2.5}$ values, where these values could possibly exist as outliers. Thus, by removing outliers in the model calculation (model 6), an increase in the coefficient estimation and/or correlation significance was possible. In general, our study findings are supported by prior studies; for example, Liu's study demonstrated $\mathrm{PM}_{2.5}$ exposure can increase the risk of experiencing pneumonia and bronchitis, the two most common lower respiratory infections ${ }^{40}$. Moreover, a study conducted in the United States reported that $\mathrm{PM}_{2.5}$ has a significant effect on the severity of health outcomes for people experiencing LRIs ${ }^{41}$. Plausible reasons for this, such as how ambient air pollutants, including $\mathrm{PM}_{2.5}$, deteriorate respiratory health, have been discussed in other studies ${ }^{42-44} \cdot \mathrm{PM}_{2.5}$ impairs the defense function of airway epithelial hosts by altering respiratory microecology and inducing immune cell dysfunction ${ }^{45}$. Further, in Yang et al.s study, they addressed how $\mathrm{PM}_{2.5}$ can damage the capacity of the immune system, particularly in the respiratory tract, which results in humans being more vulnerable to infection ${ }^{45}$.

According to the age-stratified analysis, a positive association exists between $\mathrm{PM}_{2.5}$ and LRIs across many age groups. Moreover, statistical significance was present for children (i.e., $<5$ years old and 5-14 years old) and the elderly ( $\geq 70$ years old). The effects of $\mathrm{PM}_{2.5}$ in damaging the respiratory system of children, particularly those younger than 5 years old, were supported in Egondi's study ${ }^{46}$. He concluded that high levels of exposure to outdoor $\mathrm{PM}_{2.5}$ is linked to a high child mortality rate resulting from respiratory problems, with an incidence rate ratio of $1.12(95 \% \mathrm{CI})^{46}$. Another study, this one conducted in China, revealed a significant association between $\mathrm{PM}_{2.5}$ and hospital visits for acute upper and lower respiratory infections among children younger than fifteen years old ${ }^{47}$. With a focus on children in low-middle income countries, Lelieveld et al. concluded that ambient $\mathrm{PM}_{2.5}$ accounted for approximately $5 \%$ of deaths due to LRIs and accounted for $18 \%$ of losses in life expectancy ${ }^{48}$. Compared to adults, children are much more vulnerable to air pollutants because they inhale a higher air volume per body weight than do adults and their immune systems are not yet mature ${ }^{49}$. In addition to its impact on children, $\mathrm{PM}_{2.5}$ also had an impact on the elderly, and numerous studies have provided evidence supporting the elderly to be a vulnerable group ${ }^{50-52}$.

We further investigated the association between $\mathrm{PM}_{2.5}$ exposure and LRIs in all six WHO regions. A significant positive association was found in each region (i.e., Africa, America, Europe, Southeast Asia, and the Western 
Pacific) except for the Eastern Mediterranean. A retrospective study conducted in Cameroon concluded that dry and dusty weather is a source of $\mathrm{PM}_{2.5}$ and increases the risk of acute respiratory infections in Africa ${ }^{53}$. The WHO Regional Office for Africa reported that more than $50 \%$ of premature deaths due to pneumonia among children younger than 5 years old are caused by the particulate matter ${ }^{54} \cdot \mathrm{PM}_{2.5}$ concentrations being associated with an increase in the incidence of acute lower respiratory infections has also been confirmed by several observational studies in Southeast Asia ${ }^{55,56}$, America ${ }^{57,58}$, Europe ${ }^{59,60}$, and the Western Pacific ${ }^{44,61-63}$. In a collaborative study, Burnett reported global estimates of specific mortality in terms of non-communicable diseases and lower respiratory infections were associated with long term exposure to outdoor fine particulate matter ${ }^{33}$. In this study, the only region that did not demonstrate a significant association between $\mathrm{PM}_{2.5}$ and burden of LRIs was the Eastern Mediterranean region. Khader stated that, although air pollution such as $\mathrm{PM}_{2.5}$ is recognized to be a global health problem, it is difficult to find evidence of its health effects due to the lack of data on air pollution in that region ${ }^{64}$. There are several reasons, including that the impact of air pollution on health is not perceived there to be a priority for health studies, as well as that there are shortcomings in assessing exposure-outcomes in that region. Because the Eastern Mediterranean region has different characteristics from the rest of the world not only in regard to policies for health data but also in regard to geographical conditions, this affects any exposure assessments of the region. Hence, we note this as a limitation to be considered in future studies.

Lastly, when assessing levels of greenness exposure globally, we found a positive relationship between $\mathrm{PM}_{2.5}$ and LRIs in countries with low levels of greenness as compared to countries with high levels of greenness. When focusing on analysis by region, we found significant positive associations in African, American, European, and Western Pacific countries with low levels of greenness exposure. Numerous studies have explained the protective effects of greenness exposure in decreasing $\mathrm{PM}_{2.5}$ concentrations ${ }^{65,66}$ and in reducing general health burdens and specific health burdens, such as respiratory diseases ${ }^{28,67}$. A cohort study conducted in China researched the interaction between residential greenness and mortality related to air pollution ${ }^{68}$. The study noted the synergistic effect of greenness and concluded that controlling air pollution helps to improve the public's health and wellbeing $^{68}$. Nonetheless, there were no significant associations in the Southeast Asian or Eastern Mediterranean regions regardless of greenness levels. For Southeast Asia, we suspected a significant association would not be found because the sample size was too small to conduct a stratification analysis. Faber and Fonesca previously described the phenomenon that small sample size can undermine the internal and external validity of a study and, thus, reduce statistical power ${ }^{69}$.

Several limitations should be noted. First, a country-level database may not be the most prudent area-level selection for assessments of the study variables; in the case of disease burden due to LRIs, a database is provided for the world at the city, county, and township level, so it may be wiser to consider a more granular level than the country-level in future studies. Second, this study lacks data from direct observation; and, for purposes of model development, especially for regional analyses, observational data of $\mathrm{PM}_{2.5}$ from monitoring stations is preferred because of its accuracy. Third, since this ecological study used estimates at the country-level, health data at the individual level was lacking, and that may have impacted the strength of the evidence. Fourth, several confounding variables that affect lower respiratory infections have not been considered, including genetics, race/ ethnicity, HIV status, etc. It is suggested future studies include these aforementioned confounding variables. In its spatial pattern and hotspot analyses, this study could only solve territory variation issues in terms of polygon size, and it was not able to deal with discontinuity problems for countries that did not have neighboring borders, such as Australia, New Zealand, etc. Consideration of an appropriate weight matrix is recommended for future studies. Even though some shortcomings have been presented following our findings, this study can serve as scientific evidence and a contribution to the knowledge base of critical locations of LRIs and to understand how exposure to $\mathrm{PM}_{2.5}$ can increase health problems in terms of the burden due to LRIs globally. Finally, this study offers recommendations for increasing exposure to greenness in an effort to reduce $\mathrm{PM}_{2.5}$ concentrations, which can sustainably alleviate general health burdens and specific health burdens, such as LRIs.

\section{Methods}

Lower respiratory infection database. The disability-adjusted life year (DALY) database of 183 countries was obtained for analysis from the World Health Organization (WHO) ${ }^{70}$. The estimated value of DALY due to LRIs was used to represent the burden of LRIs in each country. DALY is a summary metric of population health comprised of two metrics, namely years of life lost due to premature mortality (YLL) and years lived with disability (YLD) ${ }^{35}$. DALY represents an absolute measure of health loss by counting how many years of healthy life have been lost due to non-fatal illness, impairment, and death. The data for DALY was collected at three different levels (national, regional, and global) in four different years (2000, 2010, 2015, and 2016). Data for 183 WHO countries (Supplementary Table S1) within six WHO regions (Africa, America, Eastern Mediterranean, Europe, Southeast Asia, and Western Pacific) were extracted for this study's analysis of data at the global level (Supplementary Fig. S1). This study targets LRIs because they are a major cause globally of mortality and have a relationship with environmental exposures ${ }^{71,72}$. The International Classification of Diseases 10th revision codes (ICD-10) was used in order to identify LRIs. Pneumonia and Bronchiolitis, serving as the primary LRIs ${ }^{2}$, were identified via codes J09-J22, P23, and U04. The spatial distribution for each time frame of DALY due to LRIs is illustrated in Supplementary Fig. S2.

Fine particulate matter assessment. To estimate country-level $\mathrm{PM}_{2.5}$, we used satellite image data provided by the Atmosphere Composition Analysis Group, which was established by Prof. Randall Martin from Dalhousie University. This data has a spatial resolution of $1-\mathrm{km}$, which means that one pixel represents an area size on the ground of one-by-one kilometer. To extract the $\mathrm{PM}_{2.5}$ concentration for each country from this image, we applied the function 'zonal statistics as table' in ArcGIS and used country boundaries as a feature 
layer defining the zones. As basic information, this available $\mathrm{PM}_{2.5}$ data was assessed from satellite images via a Geographically Weighted Regression (GWR) adjustment and was processed using a validated method that combined the daily total column of aerosol optical depth (AOD) retrievals from the National Aeronautics and Space Administration (NASA) - Moderate Resolution Imaging Spectroradiometer (MODIS), Multi-angle Imaging Spectroradiometer (MISR), and Sea-viewing Wide Field-of-view Sensor (SeaWiFS) with the GEOS-Chem chemical transport model ${ }^{73}$. The data produced using this method had the advantage that it was available for all regions, and, thus, was appropriate for our study at the global level. Furthermore, in line with health outcomes being included for each of the four collection periods, information for country-level $\mathrm{PM}_{2.5}$ was accessed in 2000, 2010, 2015, and 2016. Supplementary Fig. S3 displays the geographical distribution of $\mathrm{PM}_{2.5}$ exposure for each country during each year of data collection.

Dataset of covariates. Several country-level variables that had been identified as covariates in prior studies were included in this study to investigate the association globally between $\mathrm{PM}_{2.5}$ and LRIs. This study included demographic factors that have been demonstrated to be related to health outcomes, such as population density, age, and $\operatorname{sex}^{74,75}$, and the data for these were provided by the United Nations. For investigating the effect of sex, we set male as the reference category ${ }^{76}$. Concerning socioeconomic status (SES), previous studies have indicated a strong relationship between SES and prevalence of infectious diseases ${ }^{5,77}$, such as LRIs. Data for income levels obtained from the World Bank Group were used to represent socioeconomic status. Healthcare utilization is another significant factor in health outcomes ${ }^{78,79}$. Therefore, total healthcare expenditure as the share of national Gross Domestic Product (\% of GDP) including the provision of health services (preventive and curative), family planning activities, nutrition activities, and emergency aid designated for health which provided by World Bank Group was taken into account. Lifestyle behaviors such as smoking and alcohol consumption have been demonstrated to increase the burden of LRIs ${ }^{80-82}$; accordingly, the prevalence rate of smoking and the average amount of alcohol consumption in liters per population, as provided by the World Bank Group, were controlled for in the model. Lastly, meteorological factors can trap air pollutants and further facilitate the acquisition and negative effects of respiratory diseases ${ }^{83-86}$. Therefore, we incorporated temperature data obtained from the Climatic Research Unit of the University of East Anglia-The Climate Change Knowledge Portal. Moreover, the global wind atlas (GWA 3.0), developed by Badger and his team, was also included to estimate wind speed ${ }^{87}$. It is noted that covariates were accessed during the same study periods so as to be comparable to health outcomes from the same study periods.

Analysis of spatial pattern and hotspots. Spatial statistical approaches, including spatial pattern and cluster mapping, were performed in our study. These methods are important in epidemiological studies to identify potential locations for the spread of communicable diseases such as lower respiratory infections ${ }^{15}$. These methods are common in investigating the spatial distribution of environmental exposures ${ }^{88-90}$. Using the estimated DALY adjusted for the population, the spatial autocorrelation Global Moran's I was used to assess the spatial pattern, degree of clustering, and randomness of the LRI burden globally. Moran's I is generally preferred over Geary's $C$ because the values of the former are more intuitive in measuring spatial autocorrelation ${ }^{91}$. The value of Moran's index generally varies between -1 and $1^{92}$. Positive autocorrelation occurs when similar values cluster together and a negative autocorrelation occurs when dissimilar values cluster. A value near zero means there is no autocorrelation at all. Moran's I has been found to provide a high level of performance in spatial statistical analysis and can be used to complement traditional geostatistical model ${ }^{11,12}$. This spatial analysis follows Tobler's (1970) law that "everything is related to everything else, but near things are more related than distant things." This analysis was computed with a row-standardized spatial weights matrix that was based on critical distance thresholds. Given the importance of borders in the study of global conflict, the data used was potentially biased due to sampling design and an imposed aggregation scheme ${ }^{93,94}$. A fixed distance band was then considered because it is a prudent option for data analysis when there is a large 888 variation in polygon size. The following is a Global Moran's formula,

$$
I=\frac{n \sum_{i=1}^{n} \sum_{j=1}^{n} w_{i . j} z_{\mathbf{i}} z_{j}}{s_{0} \sum_{i=1}^{n} z_{i}^{2}},
$$

where $I$ is Moran's index, $Z_{i}$ and $Z_{j}$ are the deviations for country $i$ and $j$ from the mean $\left(X_{i}-\bar{X}\right)$ and $\left(X_{j}-\bar{X}\right) ; X_{i}$ and $X_{j}$ are the numbers of DALY loss due to LRIs for country $i$ and $j$; and $X$ is average value of DALY for all countries, $W_{i j}$ is the spatial weight between $i$ and $j, n$ is equal to the total number of studied countries, and $S_{0}$ is the aggregate of all the spatial weights.

For the hotspot analysis, Getis-Ord Gi* was applied to identify the statistically significant hot spots and cold spots of burden from LRIs for all the countries (183 countries) included from the six regions. The resultant $\mathrm{z}$-scores $\left(\mathrm{Gi}^{*}\right)$ and $\mathrm{p}$-values designate the countries with either a high or low value for LRIs. The statistical significance of a $\mathrm{z}$-score assigned to each area identifies the presence and intensity of local clusters of hot spots and cold spots of LRIs relative to the hypothesis of spatial randomness. Getis-Ord Gi functions by looking at each feature within the context of neighboring values for the same feature, meaning the number of neighbors that have the same value for an indicator affects the clustering of hot spots and cold spots. If high values within $\mathrm{d}$ of other high values dominate the pattern, then the summation will result in high positive z-score values, and vice versa ${ }^{95}$; practically, this confirms a neighbor effect. In this study, hot spots were defined as the areas with the highest LRIs burden and, spatially, whose neighbors had the same high values. Meanwhile, cold spots were the areas with the lowest LRIs burden and, spatially, whose neighbors had the same low values. The Getis-Ord $\mathrm{Gi}^{\star}$ index was calculated using formula, 


$$
G_{i}^{*}=\frac{\sum_{j=1}^{n} w_{i . j} x_{j}-\chi \sum_{j=1}^{n} w_{i . j}}{s \frac{\sqrt{\left[n \sum_{j=1}^{n} w_{i . j}^{2}-\left(\sum_{j=1}^{n} w_{i . j}\right)^{2}\right]}}{n-1}}
$$

where $X_{i}$ and $X_{j}$ is number of DALY loss due to LRIs for country $i$ and $j, W_{i j}$ is the spatial weight between $i$ and $j$, and $n$ is equal to the total number of areas analyzed. Moran's I can be expressed in terms of the local $G i^{*}$ values.

Statistical model and subgroup analysis. Descriptive statistics were presented to summarize the characteristics of the variables examined in this study, and these include data of major exposures and outcomes$\mathrm{PM}_{2.5}$ and DALY loss due to LRIs, demographic factors, socioeconomic, healthcare status, behaviors, and meteorological factors. A generalized linear mixed model (GLMM) with a penalized quasi-likelihood (PQL) was applied in order to generate the study's model in investigating the association between $\mathrm{PM}_{2.5}$ and LRIs. In addition to offering a flexible approach, the GLMM was selected because this algorithm can consider both fixed and random effects in its calculation and has been applied in several environmental exposure studies related to health outcomes $^{97-99}$, as well as has widely been used in air pollution epidemiology studies ${ }^{100-102}$. For model structure, we set DALY loss due to LRIs as the dependent variable $(Y)$ and $\mathrm{PM}_{2.5}$ as the main predictor $\left(X_{1}\right)$. Several fixed effect covariates, such as demographic, socioeconomic, healthcare status, lifestyle behaviors, and meteorological factors, were further examined. The unit of the dependent variable (DALY) is "years", measured as a continuous variable, and our GLMMPQL model is based on a linear regression model. Country ID serves as the clustering unit and was treated as a random intercept and used to minimize the temporal correlation of outcomes due to repeated measurements within a country. In this case, random effect and residual error were assumed to have a multivariate normal distribution (Gaussian distribution). To model the yearly temporal variance-covariance structure, the continuous-time first-order autoregressive model, denoted AR (1), was used. Furthermore, in the case where spatial data were provided from distinct areas, in most popular implementations, a GLMMPQL can adjust the overall fixed effects while the structure of correlation remains nested within regions and allows for spatial autocorrelation only between observations in the same region ${ }^{103}$, correlation between neighbors can be included in Bayesian implementations of GLMM models. Therefore, to deal with the spatial autocorrelation problem, we also added the term of the 'continent' in the GLMMPQL calculation ${ }^{97}$. In addition, to ensure there was no multicollinearity issue across the adjusted covariates, the generalized variance-inflation factors (GVIFs) were then examined. In this study, we obtained GVIFs with a value less than four $(<4)$ for all covariates ${ }^{104}$. As a result, we included all of those GVIFs values in the Supplementary Table S2.

To investigate the robustness of our association model, we developed six sensitivity models with different covariate settings. In detail, Model 1 only controlled for demographic factors; Model 2 included demographic factors (Model 1) and the proportion of alcohol consumption; Model 3 added the prevalence rate of smoking to Model 2's inclusions; Model 4 added economic status for each country, as represented by income-level, to Model 3's considerations; and Model 5 considered healthcare expenditure in addition to Model 4's criteria. Model 6 adjusted for all covariates but excluded data from Eastern Mediterranean countries. We excluded data from Eastern Mediterranean countries in the sensitivity model to identify whether $\mathrm{PM}_{2.5}$ still remains associated with the burden of LRIs after eliminating data from countries with the highest $\mathrm{PM}_{2.5}$ (Supplementary Fig. S5); $\mathrm{PM}_{2.5}$ is highly present in Eastern Mediterranean countries because the area is naturally covered by desert dust and has low levels of vegetation ${ }^{105}$.

Subgroup analyses were also conducted in this study to determine whether the association between $\mathrm{PM}_{2.5}$ and LRIs exists within various subpopulations. Since prior studies have confirmed cases of LRIs vary by age group and, specifically, children younger than 5 years of age experience LRIs at a disproportionately high rate ${ }^{106,107}$, we conducted stratified tests for five age groups $(<5 ; 5-14 ; 15-49 ; 50-69$, and $\geq 70$ years). We also performed subgroup analyses for the six WHO regions, which include the African, American, European, Eastern Mediterranean, Southeast Asian, and Western Pacific regions. In addition, subgroup analyses were performed across regions comparing the relationship between $\mathrm{PM}_{2.5}$ and LRIs in areas with low and high levels of greenness. The greenness effect was considered in this stratified analysis because prior studies have stated that greenness can reduce the concentration of air pollutants such as $\mathrm{PM}_{2.5}$ and can directly-indirectly reduce the health burden resulting from lower respiratory infections. For the country-level greenness measurement, we used NDVI data from the Terra Moderate Resolution Imaging Spectroradiometer provided by the National Aeronautics and Space Administration with spatial resolution $1 \times 1 \mathrm{~km}^{296}$. NDVI images with the acquisition date closer to the mid-season were selected from January, April, July, and October; the selection of the months of data collection was considered for countries with two and/or four seasons. Then, we used the median of NDVI to classify the region as having a low or high exposure to greenness. Furthermore, the median values of NDVI globally and in the various regions are displayed in Supplementary Table S3. The spatial-statistical analyses were completed using ArcGIS 10.7.1 (Esri Inc., 23 Redlands, California, US) and R v. 3.6.3 developed by R Core Team ${ }^{108}$. Coefficient estimates were performed with $95 \% \mathrm{CI}$ and $\mathrm{p}$-values $<0.05$ were deemed to be statistically significant.

Received: 27 December 2019; Accepted: 16 February 2021

Published online: 01 March 2021 


\section{References}

1. Chowell, G. \& Rothenberg, R. Spatial infectious disease epidemiology: On the cusp. BMC Med. 16, 192 (2018).

2. Troeger, C. et al. Estimates of the global, regional, and national morbidity, mortality, and aetiologies of lower respiratory infections in 195 countries, 1990-2016: A systematic analysis for the Global Burden of Disease Study 2016. Lancet Infect. Dis. 18, 1191-1210 (2018)

3. Dadi, A., Kebede, Y. \& Mengesha, Z. Determinants of pneumonia in children aged two months to five years in urban areas of Oromia zone, Amhara Region, Ethiopia. OALib 01, 1-10 (2014).

4. United Nations Children's Fund (UNICEF). A fair chance for every child (accessed April 2020). http://www.soapbox.co.uk

5. Harerimana, J. M., Nyirazinyoye, L., Thomson, D. R. \& Ntaganira, J. Social, Economic and environmental risk factors for acute lower respiratory infections among children under five years of age in Rwanda. Arch. Public Health. https://doi.org/10.1186/ s13690-016-0132-1 (2016).

6. Hasegawa, K., Tsugawa, Y., Brown, D. F. M., Mansbach, J. M. \& Camargo, C. A. Jr. Trends in bronchiolitis hospitalizations in the United States, 2000-2009. Pediatrics 132, 28-36 (2013).

7. Nair, H. et al. Global and regional burden of hospital admissions for severe acute lower respiratory infections in young children in 2010: A systematic analysis. Lancet (London, England) 381, 1380-1390 (2013).

8. Murdoch, D. R. \& Howie, S. R. C. The global burden of lower respiratory infections: Making progress, but we need to do better. Lancet Infect. Dis. 18, 1162-1163 (2018).

9. Elliott, P. \& Wartenberg, D. Spatial epidemiology: Current approaches and future challenges. Environ. Health Perspect. 112, 998-1006 (2004).

10. Tsai, P.-J., Lin, M.-L., Chu, C.-M. \& Perng, C.-H. Spatial autocorrelation analysis of health care hotspots in Taiwan in 2006. BMC Public Health 9, 464 (2009).

11. Wubuli, A. et al. Socio-demographic predictors and distribution of pulmonary tuberculosis (TB) in Xinjiang, China: A spatial analysis. PLoS ONE 10, 1-22 (2015).

12. Huo, X.-N., Li, H., Sun, D.-F., Zhou, L.-D. \& Li, B.-G. Combining geostatistics with Moran's I analysis for mapping soil heavy metals in Beijing, China. Int. J. Environ. Res. Public Health 9, 995-1017 (2012).

13. Abeje, G. Prevalence of pneumonia among under- five children in Este Town and the surrounding Rural Kebeles, Northwest Ethiopia: A community based cross sectional study. Sci. J. Public Health 2, 150 (2014).

14. Abuka, T. Prevalence of pneumonia and factors associated among children $2-59$ months old in Wondo Genet district, Sidama zone, SNNPR, Ethiopia. Curr. Pediatr. Res. 21, 19-25 (2017).

15. Amsalu, E. T., Akalu, T. Y. \& Gelaye, K. A. Spatial distribution and determinants of acute respiratory infection among under-five children in Ethiopia: Ethiopian Demographic Health Survey 2016. PLoS ONE 14, e0215572-e0215572 (2019).

16. Smith, C. M. et al. Spatial methods for infectious disease outbreak investigations: Systematic literature review. Eurosurveillance. https://doi.org/10.2807/1560-7917.ES.2015.20.39.30026 (2015).

17. Tewara, M. A., Mbah-Fongkimeh, P. N., Dayimu, A., Kang, F. \& Xue, F. Small-area spatial statistical analysis of malaria clusters and hotspots in Cameroon; 2000-2015. BMC Infect. Dis. 18, 636 (2018).

18. Beck, A. F., Florin, T. A., Campanella, S. \& Shah, S. S. Geographic variation in hospitalization for lower respiratory tract infections across one county. JAMA Pediatr. 169, 846-854 (2015).

19. Tampah-Naah, A. M., Osman, A. \& Kumi-Kyereme, A. Geospatial analysis of childhood morbidity in Ghana. PLoS ONE 14, e0221324-e0221324 (2019).

20. Beamer, P. I. et al. Spatial clusters of child lower respiratory illnesses associated with community-level risk factors. Pediatr. Pulmonol. 51, 633-642 (2016).

21. Atkinson, R. W., Kang, S., Anderson, H. R., Mills, I. C. \& Walton, H. A. Epidemiological time series studies of $\mathrm{PM}_{2.5}$ and daily mortality and hospital admissions: A systematic review and meta-analysis. Thorax 69, 660-665 (2014).

22. Crouse, D. L. et al. Ambient $\mathrm{PM}_{2.5}, \mathrm{O}_{3}$, and $\mathrm{NO}_{2}$ exposures and associations with mortality over 16 years of follow-up in the Canadian Census Health and environment cohort (CanCHEC). Environ. Health Perspect. 123, 1180-1186 (2015).

23. Liu, J., Han, Y., Tang, X., Zhu, J. \& Zhu, T. Estimating adult mortality attributable to $\mathrm{PM}_{2.5}$ exposure in China with assimilated $\mathrm{PM}_{2.5}$ concentrations based on a ground monitoring network. Sci. Total Environ. 568, 1253-1262 (2016).

24. Al-Hemoud, A., Gasana, J., Al-Dabbous, N. A., Al-Shatti, A. \& Al-Khayat, A. Disability adjusted life years (DALYs) in terms of years of life lost (YLL) due to premature adult mortalities and post neonatal infant mortalities attributed to $\mathrm{PM}_{25}$ and $\mathrm{PM}_{10}$ exposures in Kuwait. Int. J. Environ. Res. Public Health 15, 2609 (2018).

25. Lim, S. S. et al. A comparative risk assessment of burden of disease and injury attributable to 67 risk factors and risk factor clusters in 21 regions, 1990-2010: A systematic analysis for the Global Burden of Disease Study 2010. Lancet (London, England) 380, 2224-2260 (2012).

26. Murray, C. J. L. et al. GBD 2010: Design, definitions, and metrics. The Lancet 380, 2063-2066 (2012).

27. Cohen, A. J. et al. Estimates and 25-year trends of the global burden of disease attributable to ambient air pollution: An analysis of data from the Global Burden of Diseases Study 2015. Lancet (London, England) 389, 1907-1918 (2017).

28. James, P., Banay, R. F., Hart, J. E. \& Laden, F. A review of the health benefits of greenness. Curr. Epidemiol. Rep. 2, 131-142 (2015).

29. Chang, A. Y. et al. Past, present, and future of global health financing: A review of development assistance, government, out-ofpocket, and other private spending on health for 195 countries, 1995-2050. Lancet 393, 2233-2260 (2019).

30. Yang, J., Mcbride, J., Zhou, J. \& Sun, Z. The urban forest in Beijing and its role in air pollution reduction. Urban For. Urban Green. 3, 65-78 (2005).

31. Dadvand, P. et al. Green spaces and general health: Roles of mental health status, social support, and physical activity. Environ. Int. 91, 161-167 (2016).

32. Shen, Y.-S. \& Lung, S.-C.C. Mediation pathways and effects of green structures on respiratory mortality via reducing air pollution. Sci. Rep. 7, 42854 (2017).

33. Burnett, R. et al. Global estimates of mortality associated with long-term exposure to outdoor fine particulate matter. Proc. Natl. Acad. Sci. 115, 9592-9597 (2018).

34. Force, L. M. et al. The global burden of childhood and adolescent cancer in 2017: An analysis of the Global Burden of Disease Study 2017. Lancet Oncol. 20, 1211 (2019).

35. Murray, C. J. L. et al. Disability-adjusted life years (DALYs) for 291 diseases and injuries in 21 regions, 1990-2010: A systematic analysis for the Global Burden of Disease Study 2010. Lancet 380, 2197-2223 (2012).

36. Zhou, M. et al. Cause-specific mortality for 240 causes in China during 1990-2013: A systematic subnational analysis for the Global Burden of Disease Study 2013. Lancet 387, 251-272 (2016).

37. Roth, G. A. et al. Global, regional, and national age-sex-specific mortality for 282 causes of death in 195 countries and territories, 1980-2017: A systematic analysis for the Global Burden of Disease Study 2017. Lancet 392, 1736-1788 (2018).

38. Fenollar, F. \& Mediannikov, O. Emerging infectious diseases in Africa in the 21st century. New Microbes New Infect. 26, S10-S18 (2018).

39. Nowbar, A. N., Gitto, M., Howard, J. P., Francis, D. P. \& Al-Lamee, R. Mortality from ischemic heart disease. Circ. Cardiovasc. Qual. Outcomes 12, e005375 (2019). 
40. Liu, Q. et al. Effect of exposure to ambient $\mathrm{PM}_{2.5}$ pollution on the risk of respiratory tract diseases: A meta-analysis of cohort studies. J. Biomed. Res. 31, 130-142 (2017).

41. Hooper, L. G. et al. Ambient air pollution and chronic bronchitis in a cohort of U.S. women. Environ. Health Perspect. 126, 027005 (2018).

42. Feng, S., Gao, D., Liao, F., Zhou, F. \& Wang, X. The health effects of ambient $\mathrm{PM}_{2.5}$ and potential mechanisms. Ecotoxicol. Environ. Saf. 128, 67-74 (2016).

43. Li, T. et al. Fine particulate matter $\left(\mathrm{PM}_{2.5}\right)$ : The culprit for chronic lung diseases in China. Chronic Dis. Transl. Med. 4, 176-186 (2018).

44. Xing, Y. F., Xu, Y. H., Shi, M. H. \& Lian, Y. X. The impact of $\mathrm{PM}_{2.5}$ on the human respiratory system. J. Thoracic Dis. 8, E69-E74 (2016).

45. Yang, L., Li, C. \& Tang, X. The impact of $\mathrm{PM}_{2.5}$ on the host defense of respiratory system. Front. Cell Dev. Biol. 8, 91 (2020).

46. Egondi, T., Ettarh, R., Kyobutungi, C., Ng, N. \& Rocklöv, J. Exposure to outdoor particles $\left(\mathrm{PM}_{2.5}\right)$ and associated child morbidity and mortality in socially deprived neighborhoods of Nairobi, Kenya. Atmosphere (Basel). 9, 351 (2018).

47. Zheng, P. et al. Air pollution and hospital visits for acute upper and lower respiratory infections among children in Ningbo, China: A time-series analysis. Environ. Sci. Pollut. Res. 24, 18860-18869 (2017).

48. Lelieveld, J., Haines, A. \& Pozzer, A. Age-dependent health risk from ambient air pollution: A modelling and data analysis of childhood mortality in middle-income and low-income countries. Lancet Planet. Health 2, e292-e300 (2018).

49. Salvi, S. Health effects of ambient air pollution in children. Pediatr. Respir. Rev. 8, 275-280 (2007).

50. Machin, A. B., Nascimento, L. F., Mantovani, K. \& Machin, E. B. Effects of exposure to fine particulate matter in elderly hospitalizations due to respiratory diseases in the South of the Brazilian Amazon. Braz. J. Med. Biol. Res. https://doi.org/10.1590/1414$431 \times 20188130$ (2019).

51. Wang, Y. et al. Long-term exposure to $\mathrm{PM}_{2.5}$ and mortality among older adults in the Southeastern US. Epidemiology 28, 207-214 (2017).

52. Yue, H., He, C., Huang, Q., Yin, D. \& Bryan, B. A. Stronger policy required to substantially reduce deaths from $\mathrm{PM}_{2.5}$ pollution in China. Nat. Commun. 11, 1-10 (2020).

53. Nsoh, M. et al. Acute respiratory infection related to air pollution in Bamenda, north west region of Cameroon. Pan Afr. Med. J. 32, 99 (2019).

54. Air Pollution | Regional Office for Africa (accessed October 2020); https://www.afro.who.int/pt/node/3828.

55. Gurley, E. S. et al. Indoor exposure to particulate matter and the incidence of acute lower respiratory infections among children: A birth cohort study in urban Bangladesh. Indoor Air 23, 379-386 (2013).

56. Reddington, C. L. et al. Exploring the impacts of anthropogenic emission sectors on $\mathrm{PM}_{25}$ and human health in South and East Asia. Atmos. Chem. Phys. 19, 11887 (2019).

57. Bowe, B., Xie, Y., Yan, Y. \& Al-Aly, Z. Burden of cause-specific mortality associated with $\mathrm{PM}_{2.5}$ air pollution in the United States. JAMA Netw. Open 2, e1915834 (2019).

58. Karr, C. et al. A case-crossover study of wintertime ambient air pollution and infant bronchiolitis. Environ. Health Perspect. 114, 277-281 (2006).

59. Lehtomäki, H. et al. Health impacts of ambient air pollution in Finland. Int. J. Environ. Res. Public Health 15, 736 (2018).

60. Willers, S. M. et al. Fine and coarse particulate air pollution in relation to respiratory health in Sweden. Eur. Respir. J. 42, 924-934 (2013).

61. Barnett, A. G. et al. Air pollution and child respiratory health: A case-crossover study in Australia and New Zealand. Am. J. Respir. Crit. Care Med. 171, 1272-1278 (2005).

62. Bennett, C. M. et al. Associations between ambient PM25 concentrations and respiratory symptoms in Melbourne, 1998-2005. J. Toxicol. Environ. Health A 70, 1613-1618 (2007).

63. Kim, K.-N., Kim, S., Lim, Y.-H., Song, I. G. \& Hong, Y.-C. Effects of short-term fine particulate matter exposure on acute respiratory infection in children. Int. J. Hyg. Environ. Health 229, 113571 (2020).

64. Khader, Y. S. Air pollution and health outcomes in the Eastern Mediterranean Region: Knowledge and research gaps and need. In Handbook of Healthcare in the Arab World (ed. Laher, I.) 1-45 (Springer, Cham, 2020).

65. Chen, M., Dai, F., Yang, B. \& Zhu, S. Effects of neighborhood green space on $\mathrm{PM}_{2.5}$ mitigation: Evidence from five megacities in China. Build. Environ. 156, 33-45 (2019).

66. Kim, S., Kim, H. \& Lee, J. T. Interactions between ambient air particles and greenness on cause-specific mortality in seven Korean metropolitan cities, 2008-2016. Int. J. Environ. Res. Public Health 16, 1866 (2019).

67. Jaafari, S., Shabani, A. A., Moeinaddini, M., Danehkar, A. \& Sakieh, Y. Applying landscape metrics and structural equation modeling to predict the effect of urban green space on air pollution and respiratory mortality in Tehran. Environ. Monit. Assess. $192,1-15(2020)$.

68. Ji, J. S., Zhu, A., Lv, Y. \& Shi, X. Interaction between residential greenness and air pollution mortality: Analysis of the Chinese Longitudinal Healthy Longevity Survey. Lancet Planet. Health 4, e107-e115 (2020).

69. Faber, J. \& Fonseca, L. M. How sample size influences research outcomes. Dental Press J. Orthod. 19, 27-29 (2014).

70. WHO |Disease burden and mortality estimates (accessed June 2019); https://www.who.int/healthinfo/global_burden_disease/ estimates/en/index1.html.

71. Zhou, M. et al. Mortality, morbidity, and risk factors in China and its provinces, 1990-2017: A systematic analysis for the Global Burden of Disease Study 2017. Lancet 394, 1145-1158 (2019).

72. McMorrow, M. L. et al. Severe acute respiratory illness deaths in Sub-Saharan Africa and the role of influenza: A case series from 8 countries. J. Infect. Dis. 212, 853-860 (2015).

73. van Donkelaar, A. et al. global estimates of fine particulate matter using a combined geophysical-statistical method with information from satellites, models, and monitors. Environ. Sci. Technol. 50, 3762-3772 (2016).

74. Amugsi, D. A. et al. Socio-demographic and environmental determinants of infectious disease morbidity in children under 5 years in Ghana. Glob. Health Action 8, 29349 (2015).

75. Falagas, M. E., Mourtzoukou, E. G. \& Vardakas, K. Z. Sex differences in the incidence and severity of respiratory tract infections. Respir. Med. 101, 1845-1863 (2007).

76. Loeb, M., McGeer, A., McArthur, M., Walter, S. \& Simor, A. E. Risk factors for pneumonia and other lower respiratory tract infections in elderly residents of long-term care facilities. Arch. Intern. Med. 159, 2058-2064 (1999).

77. Kivimäki, M. et al. Association between socioeconomic status and the development of mental and physical health conditions in adulthood: A multi-cohort study. Lancet Public Heal. 5, e140-e149 (2020).

78. Alemayehu, S., Kidanu, K., Kahsay, T. \& Kassa, M. Risk factors of acute respiratory infections among under five children attending public hospitals in southern Tigray, Ethiopia, 2016/2017. BMC Pediatr. https://doi.org/10.1186/s12887-019-1767-1 (2019).

79. Goins, W. P., Talbot, H. K. \& Talbot, T. R. Health care-acquired viral respiratory diseases. Infect. Dis. Clin. N. Am. 25, 227-244 (2011).

80. Saldias, F. \& Diaz, O. Cigarette smoking and lower respiratory tract infection. In Bronchitis (ed. Martn-Loeches, I.) (InTech, New York, 2011)

81. Mehta, A. J. \& Guidot, D. M. Alcohol and the lung. Alcohol Res. Curr. Rev. 38, 243-254 (2017). 
82. Simou, E., Britton, J. \& Leonardi-Bee, J. Alcohol and the risk of pneumonia: A systematic review and meta-analysis. BMJ Open 8, 22344 (2018).

83. Mäkinen, T. M. et al. Cold temperature and low humidity are associated with increased occurrence of respiratory tract infections. Respir. Med. 103, 456-462 (2009).

84. Liu, Y. et al. Association between temperature change and outpatient visits for respiratory tract infections among children in Guangzhou, China. Int. J. Environ. Res. Public Health 12, 439-454 (2015).

85. Liu, Y. et al. Impact of meteorological factors on lower respiratory tract infections in children. J. Int. Med. Res. 44, 30-41 (2016).

86. Zeng, S. \& Zhang, Y. The effect of meteorological elements on continuing heavy air pollution: A case study in the Chengdu area during the 2014 Spring Festival. Atmosphere (Basel) 8, 71 (2017).

87. Global Wind Atlas (accessed October 2020); https://globalwindatlas.info/about/credits.

88. Meng, H., Zhang, K., Ba, M. \& Sun, Y. Spatial autocorrelation analysis of city $\mathrm{PM}_{2.5}$ concentration in Henan. Int. J. Res. 7, 454-462 (2019).

89. Nadian, M., Mirzaei, R. \& Soltani Mohammadi, S. Application of Moran'sI Autocorrelation in spatial-temporal analysis of $\mathrm{PM}_{2.5}$ pollutant (a case study: Tehran City). J. Environ. Health Eng. 5, 197-213 (2018).

90. Zhang, H. \& Tripathi, N. K. Geospatial hot spot analysis of lung cancer patients correlated to fine particulate matter $\left(\mathrm{PM}_{2.5}\right)$ and industrial wind in Eastern Thailand. J. Clean. Prod. 170, 407-424 (2018).

91. Getis, A., Cliff, A. D. \& Ord, J. K. 1973: Spatial autocorrelation. Lond. Pion. Prog. Hum. Geogr. 19, 245-249 (1995).

92. Overmars, K., de Koning, G. H. J. \& Veldkamp, A. Spatial autocorrelation in multi-scale land use models. Ecol. Modell. 164, 257-270 (2003).

93. Anselin, L. \& Hudak, S. Spatial Data Analysis with Gis: An Introduction to Application in the Social Sciences (1992).

94. Anselin, L. Local indicators of spatial association-LISA. Geogr. Anal. 27, 93-115 (1995).

95. Getis, A. \& Ord, J. K. The analysis of spatial association by use of distance statistics. Geogr. Anal. 24, 189-206 (1992).

96. Didan, K. MOD13A3 MODIS/Terra vegetation Indices Monthly L3 Global $1 \mathrm{~km}$ SIN Grid V006. In NASA EOSDIS Land Processes DAAC. https://doi.org/10.5067/MODIS/MOD13A3.006.

97. Asri, A. K. et al. Global greenness in relation to reducing the burden of cardiovascular diseases: Ischemic Heart disease and stroke. Environ. Res. Lett. 15, 124003 (2020).

98. Casals, M., Girabent-Farrés, M. \& Carrasco, J. L. Methodological quality and reporting of generalized linear mixed models in clinical medicine (2000-2012): A systematic review. PLoS ONE 9, e112653 (2014).

99. Leung, W. T. F. et al. How is environmental greenness related to students' academic performance in English and Mathematics? Landsc. Urban Plan. 181, 118-124 (2019).

100. Chuang, Y. et al. Generalized linear mixed models in time series studies of air pollution. Atmos. Pollut. Res. 2, 428-435 (2011).

101. Szyszkowicz, M., Willey, J. B., Grafstein, E., Rowe, B. H. \& Colman, I. Air pollution and emergency department visits for suicide attempts in Vancouver, Canada. Environ. Health Insights 4, S5662 (2010).

102. Chan, T.-C., Hu, T.-H., Chu, Y.-H. \& Hwang, J.-S. Assessing effects of personal behaviors and environmental exposure on asthma episodes: A diary-based approach. BMC Pulm. Med. 19, 231 (2019).

103. Dormann, F. C. et al. Methods to account for spatial autocorrelation in the analysis of species distributional data: A review. Ecography 30, 609-628 (2007).

104. Helbich, M., de Beurs, D., Kwan, M. P., O'Connor, R. C. \& Groenewegen, P. P. Natural environments and suicide mortality in the Netherlands: A cross-sectional, ecological study. Lancet Planet. Health 2, e134-e139 (2018).

105. Querol, X. et al. Monitoring the impact of desert dust outbreaks for air quality for health studies. Environ. Int. 130, 104867 (2019).

106. Adane, M. M., Alene, G. D., Mereta, S. T. \& Wanyonyi, K. L. Prevalence and risk factors of acute lower respiratory infection among children living in biomass fuel using households: A community-based cross-sectional study in Northwest Ethiopia. BMC Public Health 20, 363 (2020).

107. Seidu, A. A. et al. Prevalence and determinants of acute lower respiratory infections among children under-five years in subSaharan Africa: Evidence from demographic and health surveys. SSM Popul. Health 8, 100443 (2019).

108. R Core Team. R: A language and environment for statistical computing. R Foundation for Statistical Computing, Vienna Austria (accessed April 2020). http://www.R-project.org

\section{Acknowledgements}

This study was supported by World Health Organization (WHO), which provided the metrics, i.e. disabilityadjusted life years (DALY) database to estimate global disease burden and mortality due to lower respiratory tract infections (LRIs) (https://www.who.int/healthinfo/global_burden_disease/estimates/en/index1.html); the National Aeronautics and Space Administration (NASA), which provided open access database for global greenness-NDVI (https://doi.org/10.5067/MODIS/MOD13A3.006); and the Atmospheric Composition Analysis Group (Aaron van Donkelaar et al. ${ }^{74}$ ) which provided global $\mathrm{PM}_{2.5}$ data (http://fizz.phys.dal.ca/ atmos/marti $\mathrm{n} /$ ?page_id=140). Moreover, this research was funded by Ministry of Science and Technology (MOST), Grant Number 108-2621-M-006-071.

\section{Author contributions}

Conceptualization, C.-D.W., H.-J.S. and J.D.S.; Methodology, C.-D.W., W.-C.P. and A.K.A; Formal analysis, A.K.A., H.-Y.L. and C.-D.W; Writing-original draft preparation, A.K.A.; Writing-review and editing, W.-C.P., C.-D.W., H.-Y.L. and A.K.A.; Supervision, C.-D.W., W.-C.P., J.D.S. and H.-J.S.; Funding acquisition, C.-D.W. and H.-J.S.

\section{Competing interests}

The authors declare no competing interests.

\section{Additional information}

Supplementary Information The online version contains supplementary material available at https://doi. org/10.1038/s41598-021-84435-y.

Correspondence and requests for materials should be addressed to C.-D.W.

Reprints and permissions information is available at www.nature.com/reprints. 
Publisher's note Springer Nature remains neutral with regard to jurisdictional claims in published maps and institutional affiliations.

(c) (i) Open Access This article is licensed under a Creative Commons Attribution 4.0 International License, which permits use, sharing, adaptation, distribution and reproduction in any medium or format, as long as you give appropriate credit to the original author(s) and the source, provide a link to the Creative Commons licence, and indicate if changes were made. The images or other third party material in this article are included in the article's Creative Commons licence, unless indicated otherwise in a credit line to the material. If material is not included in the article's Creative Commons licence and your intended use is not permitted by statutory regulation or exceeds the permitted use, you will need to obtain permission directly from the copyright holder. To view a copy of this licence, visit http://creativecommons.org/licenses/by/4.0/.

(C) The Author(s) 2021 